\title{
Equidad educativa en Chihuahua en cifras: 1900-1954
}

\author{
Guillermo Hernández Orozco \\ Universidad Autónoma de Chihuahua \\ ghernand@uach.mx \\ Martha Esther Larios Guzmán \\ Universidad Autónoma de Chihuahua \\ marthalarios guzman@yahoo.com.mx
}

\begin{abstract}
Resumen
La inserción de la mujer en el mercado laboral y el reclamo de la igualdad de derechos y obligaciones de la mujer, subyugada al poder varonil por centurias, trajo en el mundo la necesidad de conformar una cultura igualitaria. Proceso nada sencillo, que dentro de su complejidad históricamente se ha venido construyendo de manera asimétrica entre las diferentes regiones del país. Aquí se trata de ofrecer algunos elementos de ese andamiaje político-académico y cultural desde la perspectiva estadística.
\end{abstract}

Palabras clave

Historiografía, educación, Chihuahua.

\section{Introducción}

La identidad regional se construye con los entramados de diversas variables como: creencias religiosas, costumbres familiares, aspiraciones sociales, grupo social de pertenencia, tiempo histórico, condición económica, educación escolar y hasta latitudes geográficas. Un factor importante es la posición que juega la mujer en la construcción de su propia sociedad. El período que se analiza comprende de 1900 a 1954, ya que al inicio del siglo XX la mujer Chihuahua visiblemente gana espacios educativos, con la creación reciente de la Escuela Industrial para Señoritas, la Escuela Comercial en 1904 y la Escuela Normal en 1906. Para 1954 hay un reacomodo de la matrícula al transformarse el Instituto científico y Literario en universidad.

Este trabajo está realizado a partir de fuentes primarias, todas concentradas en el Archivo Histórico de la Universidad Autónoma de Chihuahua (AHUACH): correspondencia, informes de los directores, estadística básica que se emitía, listas de asistencia del profesorado, libros de inscripciones y asistencia de alumnos.

\section{Desarrollo}

Chihuahua más que otras entidades de la República, favoreció la educación de las mujeres debido quizá a la cercanía con la nación del norte y de donde nos llegaron grandes educadores que implantaron escuelas como el Palmore, el Anáhuac y todos aquellos que establecieron en centros mineros. Hubo Colegios como el alemán o el establecido en la Hacienda Humbolt, en el municipio de Julimes con los inmigrantes de África del Sur, con el grupo Boer (Periódico Oficial del Gobierno del Estado de Chihuahua, 1906). 
Según el informe de gobierno de 1906 había inscritos en educación primaria 12 428 hombres y 7630 mujeres, donde aún se percibe desigualdad en la cantidad de hombres y mujeres que recibían educación. Para 1908 los datos más desglosados indican que recibían educación en primaria elemental 9685 niños y 7618 niñas, mientras que, en la educación primaria superior, es decir el 5o. Y 6o. Grado, hubo 1334 niños y 1325 niñas. Prácticamente era la mitad de cada género (Creel, 1909).

En el caso del Instituto Científico y Literario, en 1906 en la Escuela Preparatoria había 26 hombres inscritos y ninguna mujer. Los estudiantes de Preparatoria tenían la intención de seguir carreras profesionales, las que necesariamente se implicaba trasladarse a México o Estados Unidos, lugares de los más frecuentes. No así a El Paso donde aún no había universidad. En ese año de 1906 en cambio se inscribieron en la Escuela Normal 10 mujeres y ningún hombre y en lo que se refiere a la Escuela Comercial los alumnos sumaban 29 y 10 las mujeres, cifras que pronto se revertirían (Archivo Histórico de la Universidad Autónoma de Chihuahua [AHUACH], 1892).

En el año inicio de la Revolución, en 1910 en la Escuela Preparatoria se inscribieron 30 hombres y aparecen en las estadísticas 5 mujeres. Una de ellas es María Brizándes Marín que 3 años después ingresa a Medicina en la UNAM, siendo la primera mujer que estudió en el ICL y que aparece en dicha carrera. Por lo que se refiere a la Escuela Normal en ese año se inscribieron 13 hombres y 50 mujeres, por su parte en la Escuela Comercial son 29 mujeres las inscritas y 10 hombres.

En 1912 se desata un escándalo en el ICL debido a que el periódico "El País" de la capital de la república publica un artículo dando cuenta de que en el Instituto ha educación mixta. Para proceder a las aclaraciones pertinentes se reúne la planta de maestros y la coeducación que ellos consideraban normal, en el sur del país no lo era. Ante la pregunta del director que desde cuándo se había iniciado en el Instituto, no recuerdan bien la fecha y se limitan a decir que desde que se acuerdan, la realidad es que en 1892 se inscribió la primera mujer en el Instituto, ella fue Francisca Beltrán del Río, luego le siguió María Velarde y otras 5 antes de finalizar el siglo. Los maestros elaboran un comunicado y se manda publicar en periódicos de Distrito Federal como "Nueva Era" y "El País", Tomás Berlanga es el encargado de que se publiquen (AHUACH, 1912).

Dos años después en 1914 se publica la Ley Reglamentaria de la Escuela Normal Mixta la cual no deja lugar a dudas de que Chihuahua se adelanta normativa y realmente a impulsar la educación vista desde la igualdad de género (Periódico Oficial del Estado de Chihuahua, 1912).

Los próximos años son prolijos en estadísticas que especifican los hombres y las mujeres que estudiaron en el Instituto, así en 1917 en la Preparatoria hay 23 alumnos y ninguna mujer, mientras que en la Escuela Normal solo hay 4 hombres y 53 mujeres, por su parte la Escuela Comercial inscribe a 38 hombres y 45 mujeres. Aun predominaba en el ICL mayor número de hombres. Se estaba oficialmente en el año de la terminación de la Revolución.

$\mathrm{Al}$ año siguiente de 1918 se iniciaba un largo, larguísimo periodo de promesas y esfuerzos reales por materializar las utopías y esperanzas de la Revolución, una de ellas es que habría más impulso a la educación, discurso que en la realidad no se podrá alcanzar en los siguientes 20 años, al menos las cifras así lo muestran. La 
equidad en la educación también será un discurso reiterativo. En el año de 1919 se inscribieron en el ICL: en Preparatoria 67 hombres y ninguna mujer, mientras que en la Normal ningún hombre y 62 mujeres, por su parte en la otra Escuela del Instituto, la Comercial la matrícula es de 35 hombres y 99 mujeres (AHUACH, 1929). Es la primera vez que en el ICL hay inscritos más mujeres que hombres. Esta será una de las razones por las cuales, con el tiempo, el grupo de poder del ICL representado por los hijos de los que estudiaban la Preparatoria, acaba por separar a las otras dos escuelas. La Comercial en 1924 y la Normal en 1937.

La inscripción en el ICL para 1920 fue de 69 hombres en la Preparatoria y ninguna mujer, mientras que en la Normal fueron sólo 2 hombres y 70 mujeres, por su parte en la Escuela Comercial hubo 29 hombres y 88 mujeres. En cuanto a titulados sólo aparecen de la Escuela Normal, fueron 8 mujeres y ningún hombre.

Los titulados de la Escuela Normal nos muestran el grado de equilibrio existente entre hombres y mujeres. En 1921 son 9 hombres e igual número de mujeres. Para 1922 no se titula ningún hombre y lo hacen 6 mujeres. Al año siguiente de 1923 aparece un varón titulado y 7 mujeres. La desproporción aumenta para 1924 cuando se titula un hombre y 10 mujeres. En 1925 sucede algo similar, hay 3 hombres titulados y 11 mujeres. Continúa esa proporción en 1926 cuando se titula un hombre y 9 mujeres. Al año siguiente no hay hombres titulados y sí 5 mujeres.

Entre 1920 y 1929 el total de titulados en

\footnotetext{
1 En 1922 se decretó la supresión de la Escuela Comercial y las nuevas alumnas debían inscribirse en la Escuela Industrial para Señoritas. Por dos años más permaneció
}

la Escuela normal es de 20 hombres y de 91 mujeres. Está clara la proporción de más de 4 por 1 lo que indica el fuerte proceso de feminización de la profesión magisterial, pero también se señala como el gran espacio laboral de la mujer, desde luego que prioritariamente en el ámbito educativo.

En cuanto a la inscripción de hombres y mujeres en el ICL entre 1926 y 1932 se muestra que en 1926 en la Escuela Preparatoria hubo 114 hombres y 5 mujeres, mientras que en la Escuela Normal fueron 31 hombres y 131 mujeres. En la Escuela Comercial en liquidación aún quedaban 35 hombres y 99 mujeres. ${ }^{1}$ Para 1928 en la Preparatoria se inscriben 173 hombres y 23 mujeres, cantidad altísima si se consideran los años anteriores. Por su parte en la Escuela Normal se matriculan 55 hombres y 107 mujeres.

El año de 1929 es particularmente importante para la educación en Chihuahua, ya que se inicia la Escuela Normal de Educadoras y la Normal Nocturna se integra al ICL, igualmente se designa como Escuela Secundaria para los alumnos que venían estudiando 3 años después de la primaria superior. Ese año se inscriben en la Preparatoria 150 hombres y 26 mujeres, en cuanto a la Escuela Normal Diurna 46 hombres y 131 mujeres, mientras que en la Normal Nocturna la matrícula es de 37 mujeres y ningún hombre. La Escuela Normal de Educadoras inscribe en su primera generación a 35 mujeres y lógicamente ningún hombre.

En el año de 1931 la planta docente está integrada por 10 hombres y 1 mujer en la Preparatoria. En la Escuela Normal

funcionando la escuela, hasta que terminaron sus estudios quienes se encontraban matriculadas. 
Diurna son 34 maestros y 14 maestras, mientras que en la Escuela Normal Nocturna fueron 8 profesores y 7 profesoras, en cuanto a la Escuela normal de Educadoras se contaba con 2 hombres y 5 mujeres (AHUACH, 1931).

La matrícula de 1932 muestra que en la Escuela Preparatoria hubo 167 hombres y 13 mujeres. En la Escuela Normal Diurna fueron 27 hombres y 160 mujeres. Por lo que se refiere a la Escuela Normal Nocturna fueron 7 hombres y 16 mujeres, mientras que en la Escuela Secundaria fueron 201 hombres y 235 mujeres.

Como puede apreciarse el número de inscritos en el Instituto Científico y Literario, era mayor el de mujeres que el de hombres.

La conformación del ICL de Chihuahua de 1934 a 1954 se concreta en los alumnos inscritos en cada una de sus Escuelas: Normales (Diurna y Nocturna), de Ingenieros Mecánicos y Electricistas, Topógrafos Industriales, Farmacia, Secundaria, Prevocacional, Vocacional y Preparatoria. Desde la inscripción por género ¿cuál fue el comportamiento? Veamos algunas cifras.

En 1933 la matrícula de la Escuela Secundaria fue de 315 de los cuales dominaron los hombres quienes fueron 173 y mujeres 142. Mientras que en la Escuela Preparatoria preponderantemente eran hombres, es decir 64 por 26 mujeres, no así en la Escuela Normal donde la inscripción fue de 10 hombre por 51 mujeres en la Diurna y 6 varones por 23 mujeres. En cuanto a la Escuela de Ingenieros Mecánicos y Electricista sólo hubo alumnos varones: 44. Es decir 297 hombres y 242 mujeres. Aunque son más hombres que mujeres, para la época resulta sumamente interesante considerar casi el equilibrio de matrículas.

El informe presentado por el director del ICL correspondiente a 19331934 señala que en la Escuela Secundaria hay inscritos 179 hombres y 142 mujeres, es decir, prácticamente es una matrícula equilibrada, mientras que en la Escuela Normal únicamente son 10 hombres y 51 mujeres, en cuanto a la Escuela Preparatoria el proceso es inverso, se inscribieron 45 hombres y solamente 1 mujer, mientras que la Escuela de Ingenieros Mecánicos y Electricistas son 53 hombres y ninguna mujer. En total 303 hombres y 194 mujeres (AHUACH, 1936).

Para el ciclo escolar 1934-1935 las inscripciones por sexo en las escuelas del Instituto Científico y Literario fueron: en la Escuela Secundaria 186 hombres por 149 mujeres, mientras que en la Escuela Preparatoria la inscripción de hombres fue de 30 hombres y ninguna mujer, en cuanto a la Escuela Normal se matricularon 13 hombres y 59 mujeres, por su parte en la Escuela de Ingenieros Mecánicos y Electricistas la inscripción fue de 73 estudiantes, todos hombres. En total 302 hombres y 208 mujeres. Como puede apreciarse la relación de estudiantes es de $60 \%$ de hombres por el $40 \%$ de mujeres (AHUACH, 1935).

En cuanto a la planta del profesorado, considerando el género, fue la siguiente: en 1933, en la Escuela Normal 10 profesores y 2 profesoras. En la Escuela Preparatoria hubo 7 profesores, por lo que se refiere a la Escuela de Ingenieros Mecánicos y Electricistas la planta de maestros estuvo integrada por 6 hombres y una mujer. En total 23 hombres y sólo 3 mujeres.

Al año siguiente, 1934, el profesorado estuvo integrado en la Escuela Secundaria por 24 hombres y 11 mujeres. La Escuela Normal por 12 
profesores y 5 profesoras. La Preparatoria por 7 hombres y ninguna mujer, por su parte la EIME contó con 13 profesores y una profesora. En total 56 hombres y 17 mujeres (AHUACH, 1934).

En 1935 la matrícula de la Escuela Secundaria comprendió 189 hombres y 149 mujeres, mientras que en la Preparatoria se inscribieron 30 hombres y ninguna mujer, por su parte en la Normal se matricularon 13 y 59 mujeres, en cuanto a la Escuela de Ingenieros Mecánicos y Electricistas hubo una inscripción de 73 alumnos y ninguna alumna. En cuanto al profesorado estaba integrado en la Escuela Secundaria por 24 hombres y 11 mujeres. En tanto que en la Escuela Normal fueron 12 profesores y 5 profesoras, por su parte en la Escuela de Ingenieros Mecánicos y Electricistas impartieron clases 13 hombres y una mujer, en cuanto a la Preparatoria sólo trabajaron 7 hombres. La Escuela de Ingenieros topógrafos aparece por primera vez con una inscripción de 4 estudiantes todos varones. En síntesis, en ese año el total de la matrícula de las escuelas del ICL fue de 306 alumnos y 208 alumnas, atendidos por 56 maestros y 17 maestras.

Los datos estadísticos de 1936 del ICL muestran que en la Escuela Secundaria se inscribieron 130 mujeres y no aparecen las cifras relativas a los alumnos de las demás escuelas. Ciertamente en ese año, más que escuela funcionó la Cátedra de Derecho Obrero, con una matrícula oficial de 34 estudiantes a los cuales no se les pedía antecedentes escolares sino militancia sindical, pero los datos no señalan cuantos fueron hombres y cuantas mujeres, aunque se presume que casi todos eran varones. Para 1937 en la Escuela Secundaria se matricularon 144 varones por sólo 40 mujeres, cifra que parece sumamente disminuida, si consideramos las estadísticas relativas a los años anteriores. En la Escuela Preparatoria fueron 26 hombres por 7 mujeres las inscritas. En cuanto a la Normal la inscripción fue de 47 alumnos y 120 alumnas, este fue el último año que esta escuela estuvo integrada al ICL, mientras que en la Escuela de Ingenieros Mecánicos y Electricistas, la inscripción fue siempre de varones, en este año comprendió 83 alumnos, la cifra más alta encontrada en toda su vida académica. Por su parte la Escuela de Ingenieros Topógrafos mantuvo su pobre inscripción de 3 alumnos varones.

La matrícula encontrada de la Escuela Secundaria sólo de sexo femenino fue en 1938 de 54, mientras que la Escuela de Ingenieros Topógrafos matriculó a 9 varones. Para el año siguiente se matricularon 58 varones en la Escuela Secundaria y 12 en la de Ingenieros Topógrafos. En 1940 los datos muestran que en el ICL se inscribieron en la Escuela Secundaria 170 hombres y 44 mujeres, la Preparatoria matriculó a 40 varones y 7 alumnas, por su parte la Escuela de Ingenieros Mecánicos y Electricistas inscribió a 74 estudiantes hombres y la Escuela de Ingenieros Topógrafos aparece con una raquítica matrícula de 9 varones. En el año de 1942 en la Escuela Secundaria se inscribió a 230 hombres y 64 mujeres, para 1944 las cifras encontradas muestran que en esa misma escuela la matrícula fue de 76 varones y 15 mujeres y por primera vez aparecen los datos respectivos de la Escuela Prevocacional con 55 hombres y 2 mujeres, igualmente se desagrega la matrícula de la Escuela Vocacional, cuya inscripción fue de 9 todos varones. Por su parte la Escuela Preparatoria reporta 124 hombres y 22 mujeres. Los datos muestran a lo largo de los años que para las agrupaciones estadísticas se cambiaba de criterio constantemente. 
Para 1947 las estadísticas muestran que en la Escuela Secundaria se inscribieron 46 alumnas sin que se haya encontrado el dato de alumnos, mientras que en la Escuela Prevocacional tampoco aparecen cifras relativas a la matrícula, por su parte en la Escuela Vocacional fueron 13 alumnos y ninguna alumna, en cuanto a la Preparatoria fueron 192 los alumnos y 38 las alumnas. Llama la atención el aumento desmedido de las cifras en esta última escuela.

En 1950 al parecer las cifras no están desagregadas por escuelas y se muestran como de la Preparatoria, con una matrícula de 229 hombres y 82 mujeres sólo para la escuela diurna mientras que para la Preparatoria Nocturna son 41 varones los inscritos y 3 mujeres. Aparecen por primera vez los datos de la Escuela de Farmacia con una matrícula de 27 hombres y 63 mujeres.

Un año después, en 1951 los datos arrojan que se inscribieron en la Escuela Preparatoria Diurna 274 hombres y 59 mujeres, mientras que en la nocturna fueron 27 varones y 5 alumnas. Por su parte en la Escuela de Farmacia fueron 39 alumnos y 73 alumnas.

Para 1953 de nuevo aparecen desagregados los datos de la Escuela Prevocacional con una inscripción de 151 hombres y 13 mujeres, mientras que la Escuela Preparatoria diurna arroja cifras de 304 hombres y 40 mujeres, en cuanto a la Preparatoria nocturna se inscribieron 33 estudiantes, todos varones. En la Escuela de Farmacia se matricularon 51 hombres y 128 mujeres.
En síntesis, en la Escuela Secundaria había alta matrícula de mujeres, es decir entre un 30 y $40 \%$, mientras que las escuelas Normal y de Farmacia predominaban la mujeres, en promedio entre un 70 y $75 \%$, mientras que las escuelas Prevocacional, Vocacional, Preparatoria, de Ingenieros Mecánicos y Electricistas como la de Ingenieros Topógrafos lo fueron eminentemente de varones.

\section{Referencias}

AHUACH. (1892). Libro de inscripciones.

AHUACH. (1912). Correspondencia de fecha 30 de agosto de 1912.

AHUACH. (1929). Informe del director.

AHUACH. (1931). Libro de asistencia del personal académico $y$ administrativo del ICL.

AHUACH. (1934). Informe del director del ICL correspondiente al año de 1934.

AHUACH. (1935). Informe del director del ICL.

AHUACH. (1936). Informe correspondiente a 1932 a 1936 del director Francisco García Carranza de fecha 1 de octubre de 1936.

Creel, Enrique (1909). Informe de Gobierno de fecha 1 de junio de 1909. AHUACH.

Periódico Oficial del Estado de Chihuahua. (1906-9). Archivo Histórico Universitario (AHUACH).

Periódico Oficial del Estado de Chihuahua. (1914, diciembre 31). AHUACH. 